Nigerian Journal of Environmental Sciences and Technology (NIJEST)

www.nijest.com

ISSN (Print): 2734-259X ｜ＩSSN (electronic): 2734-2603

Vol 5, No. 2 October 2021, pp 320 - 328

\title{
Designing a Spectrum Allocation Chart for Nigeria
}

\author{
Bello N. ${ }^{1 *}$ and Edeko F. O. ${ }^{2}$ \\ Department of Electrical and Electronic Engineering, Faculty of Engineering, University of Benin, Benin City, \\ Nigeria \\ Corresponding Author: *nosabello@uniben.edu
}

https://doi.org/10.36263/nijest.2021.02.0277

\begin{abstract}
The regulation of the radio spectrum today by government agencies addresses the issue of interference between radio stations by allocating, allotting and registering bands of radiofrequencies. The framework of management is done at the international, regional and national level. With this paper, we present a study of the radio spectrum allocation policies in some leading countries and Nigeria. However, narrowing the study down to the detailed design of spectrum allocation charts. The study used the information in the national table of frequency allocation (NTFA) to design the spectrum allocation chart of Nigeria with Photoshop application. The spectrum allocation chart was designed with high resolution for high zoom capabilities so that researchers can gain a quick overview of the radio services allocated in the radio spectrum in Nigeria.
\end{abstract}

Keywords: ITU, NTFA, spectrum allocation chart, frequency allocation chart, spectrum chart, NCC

\subsection{Introduction}

The radio-frequency (RF) spectrum is a natural resource; it is commonly agreed that wireless telecommunications need regulation at the national, regional and global levels. The RF spectrum is a national limited resource, much like water, land, gas and minerals. Like these, it is scarce; however, the RF is renewable, extends to higher frequencies and is not nearing exhaustion (Mazar, 2016). Optimal usage is achieved through standards at the international, regional, and national level. For instance, at the national level, a national frequency allocation table defines the categories of RF users, which is very useful to all RF users, including importers. A public allocation table permits any potential user of the spectrum to check an up-to-date database of spectrum availability, and to identify who is responsible for agreeing terms for the use of such a spectrum. Two leading English-speaking countries leading the world have allocation charts, including South Africa, New Papua Guinea, etc. Nigeria is still without one though there is a national table of frequency allocation (NTFA) accessible in the National Communication Commission (NCC) page. The Nigerian allocation chart presented is beneficial for an overview of the NTFA available in Commission \& others (2021) and useful for public consumption.

Section 3 reviews the standard for spectrum management at the three levels taking the UK and US as case studies. The material used and the methodology used in designing the spectrum chart is introduced in detail in Section 4. Section 5 discussed the spectrum chart and finally, some conclusions are drawn in Section 6.

\subsection{Spectrum management standards}

Spectrum planning is relevant towards the allocation of spectrum resource to the greatest extent possible and forecasting of future conditions of the spectrum as well as that of methods for adjusting spectrum use over time. It is the consideration of the impact of growing demand, technological innovation, and new services or improvements to existing services and the impact on planned changes to spectrum allocations. It is done at the international, regional, and national level (Ancans, et al., 2013). 
At the international level, spectrum planning is done by International Telecommunication Union (ITU) - an intergovernmental (member states) organization for the development of telecommunications in which the private sector (sector members, associates, and academia) entities participate as well. ITU is the leading United Nations agency for information and communication technologies and spans three core sectors: ITU-R - the radiocommunication sector; ITU-D - the development sector; and ITU-T - the standardization sector (Ancans, et al., 2013). The ITU-R coordinates the use of spectrum on an international level, seeking to globally harmonize radio frequency spectrum bands and to reduce harmful interference between stations of different countries to improve the use of Radio Frequency (RF) services (Union, 2021).

Spectrum management at a regional level is done by several regional organizations - e.g., the AsiaPacific Telecommunity (APT), Arab Spectrum Management Group (ASMG), African Telecommunications Union (ATU), European Conference of Postal and Telecommunications Administrations (CEPT), Inter- American Telecommunication Commission (CITEL), Regional Commonwealth in the field of Communications (RCC). For instance, in Europe region it is done by the CEPT committee, the Electronic Communications Committee (ECC). The committee brings together 48 countries to develop common policies and regulations in electronic communications and related applications for Europe as well as to provide the focal point for the information on spectrum use. One of its primary objectives is to harmonize the efficient use of radio spectrum resources across Europe. It plays an active role at the international level, preparing common European proposals to represent European interests in the ITU and other international organizations.

At a national level, spectrum management and planning are done by an appointed governmental authority (also known as Administration). Each country has its National Regulatory Authority (NRA), which is responsible for spectrum planning at the national level.

\subsection{Spectrum Management in some leading countries}

In this section we review the spectrum management in two leading English-speaking countries, the UK and the USA.

\subsubsection{Radio spectrum regulation in the UK}

The UK is part of Europe, within the CEPT group and a Member State of the European Union (EU). The management of the radio spectrum is split between the government and Ofcom. Long before the body, Ofcom, the independent spectrum regulator in the UK, formed in 2003, all radio-frequencies were managed by the government, with the Radiocommunications Agency (RA) responsible for civil use and the Ministry of Defence (MOD) responsible for military use. The MOD continues to manage the spectrum which is designated primarily for military use (Doyle \& Vick, 2005). The Communications Act 2003 passed most of the responsibilities of the RA to Ofcom, except for spectrum used by Crown bodies for which no license had ever been required as a result of Crown immunity (Mazar, 2016). Ofcom is directed in its activities by government policy.

Some of the main players in the UK regulation administration are the UK Spectrum Strategy Committee, UKSSC and the Executive Ministerial Branch for Telecommunication (Mazar, 2016). The UKSSC is the main body that oversees the development and delivery of this strategy for the government; UKSSC coordinates the organizations that have interests or holdings in the public sector spectrum and coordinates the government's policy on spectrum use. The executive ministerial branch for telecommunication is the UK government framework. It comprises departments like the Department for Business, Innovation and Skills (BIS), the Department for Culture, Media, and Sport (DCMS) and the Office of Communications (Ofcom). The BIS is responsible for maximizing the effectiveness of collaboration both with the European Union and internationally. The DCMS is packed with the responsibility for culture and sport in England, and some aspects of the media throughout the whole UK, such as broadcasting and the Internet. It also has responsibility for the tourism, leisure and creative industries (some jointly with the Department for Business, Innovation and Skills). DCMS is also responsible for the building of a digital economy. 


\subsubsection{Radio spectrum regulation in the USA}

The legal framework of the USA is formed by the Congress, the states and the Federal Courts. Congress created Federal Communications Commission (FCC) to oversee and regulate these industries. Then it adopted the Communication Act of 1934 which is periodically updated (Commission \& others, 2021). The decisions of FCC can be overridden by Congress and is reviewed by the Federal Courts. FCC is the primary authority in the United States for communications law, regulation and technological innovation. The FCC mainly takes a regulatory stance and also implements standardization functions. The FCC adopts standards developed by the technical committees and makes them regulations (Commission \& others, 2021). In the US, the main players in radio spectrum management are the NTIA, National Telecommunications and Information Administration and the Federal Communications Commission (FCC). NTIA is the executive branch agency that is principally responsible for advising the president on telecommunications (domestic and international) and information policy issues (Data, 1994). Regarding wireless communications, NTIA's programs and policy-making focus largely on expanding the use of the spectrum by all users. This goal also addresses many of the nation's most pressing needs, such as improving education, health care, and public safety (DAU, 2013).

\subsubsection{Spectrum management in Nigeria}

The Government agency in charge of the management of the radio frequency spectrum is the Nigeria communication commission (NCC) which was created under decree number 75 by the federal military government of Nigeria on the 24th of November 1992. The decree has since then been revoked and replaced with the Nigerian communication acts (NCA) which came about in 2003 (Uzairue, et al., 2018; Nigerian Communications Commission, 2021).

The agency was created to be a subsidiary of the Federal ministry of communication in Nigeria and is a member of the international government organization ITU. Nigeria became a member state of ITU on the 11th of April 1961 and has since then participated in ITU programs around the globe (Bulmer, 2020).

As of 2003, after the replacement of the degree number 75 by the federal military government of Nigeria, the NCC was empowered to make and publish regulations on matters such as permits, assignments and written authorizations, develop comprehensive frequency management policies, conduct studies and produce reports on the telecommunication industry set up a uniform numbering scheme to be used by operators to set up calls, etc. and this has helped in the substantial growth of communication in Nigeria over the years (Uzairue, et al., 2018; Regulations, 2021).

\subsubsection{National table of frequency allocation (NTFA)}

A national table of frequency allocations provides the basis for an effective and transparent spectrum management process. The international table of frequency allocations is the basis for a national table (Mazar, 2016). However, the ITU table usually allocates RF to a number of different services in a frequency band. Therefore, an administration may wish to adopt its own national table to facilitate spectrum use within its borders. For example, some countries divide their national table into bands allocated to the government and those allocated to private users.

The NTFA is the published outcome of national spectrum planning. It is the basis for an adequate spectrum management process due to some reasons. It provides a broad plan for the use of spectrum and the basic structure that will ensure effective use of the spectrum and also the prevention of radio frequency interference between services. Also, it provides a form of advice to manufacturers about where in the spectrum they should design and build equipment and to users as to what frequencies are available to plan their systems. Each NRA develops and maintains its own NTFA for the frequency range from $9 \mathrm{kHz}$ up to $3000 \mathrm{GHz}$ (or the highest planned frequency) for allocations to radiocommunication services defined by the ITU (Ancans, et al., 2013).

\subsubsection{Allocation tables in the UK and USA}

The UK frequency allocation table (FAT) regulates all the allocations of the UK spectrum from 8.3 $\mathrm{kHz}$ through to $275 \mathrm{GHz}$ (DCS, 2014; Mazar, 2016). FAT contains details of the usage of various 
frequency bands in the UK, and bodies that are responsible for planning and managing them, and making frequency assignments for civil use at particular locations. The FAT also shows the internationally agreed spectrum allocations of the ITU Radio Regulations. Ofcom published the FAT used by Research (2018) to produce the spectrum allocation chart and it is shown in Figure 1.

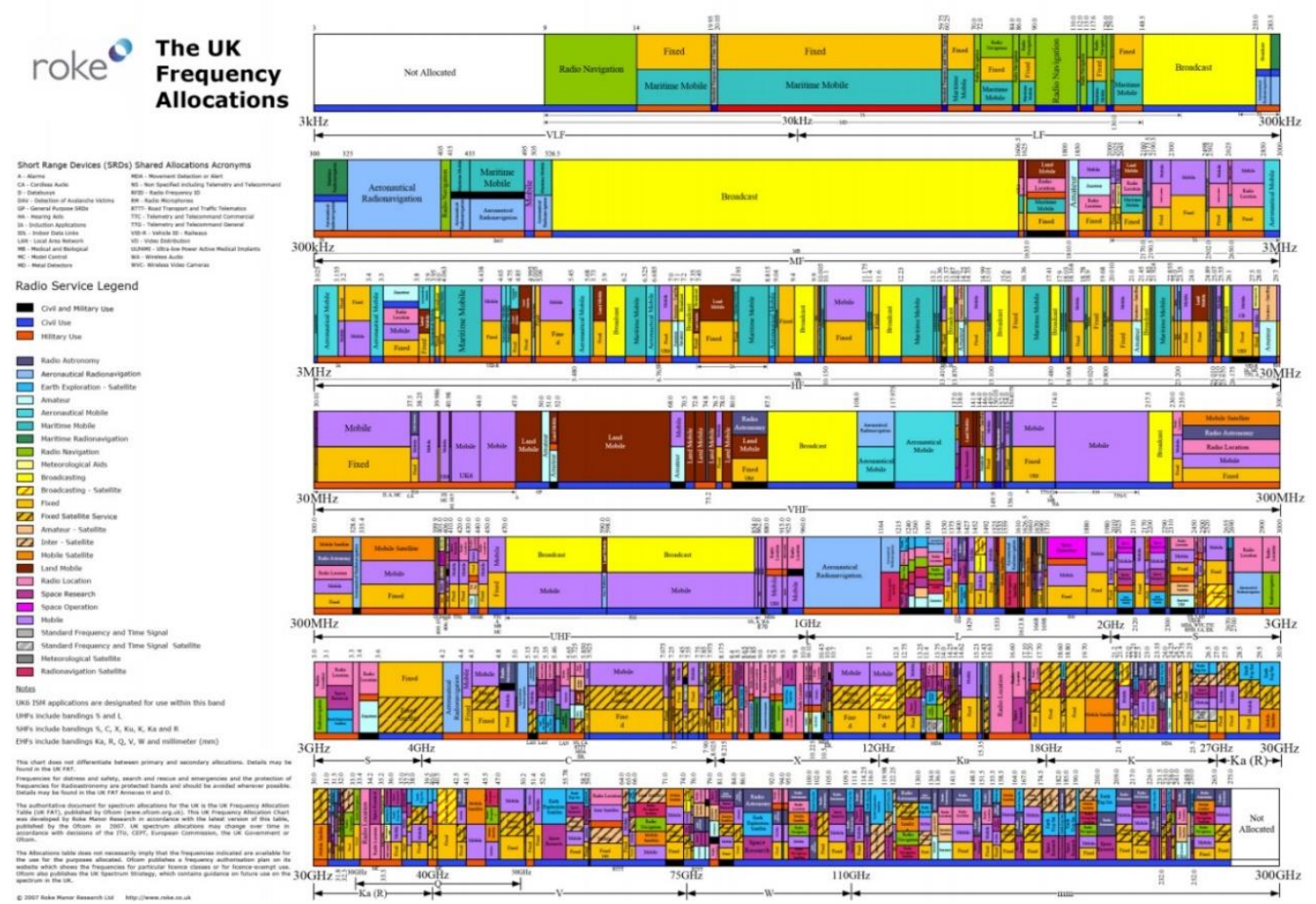

Figure 1: UK frequency allocation chart (Extreme Tech, 2021)

The NTFA of USA is presented as the FCC online table of frequency allocations regulates the radio spectrum in the US from $8.3 \mathrm{kHz}$ to $300 \mathrm{GHz}$. NTIA in the year 2011, prepared the US allocation chart (Telecomm \& Admin, 2011) shown in Figure 2. Allocation charts are designed for public consumption and gives a quick overview of the use of the radio spectrum.
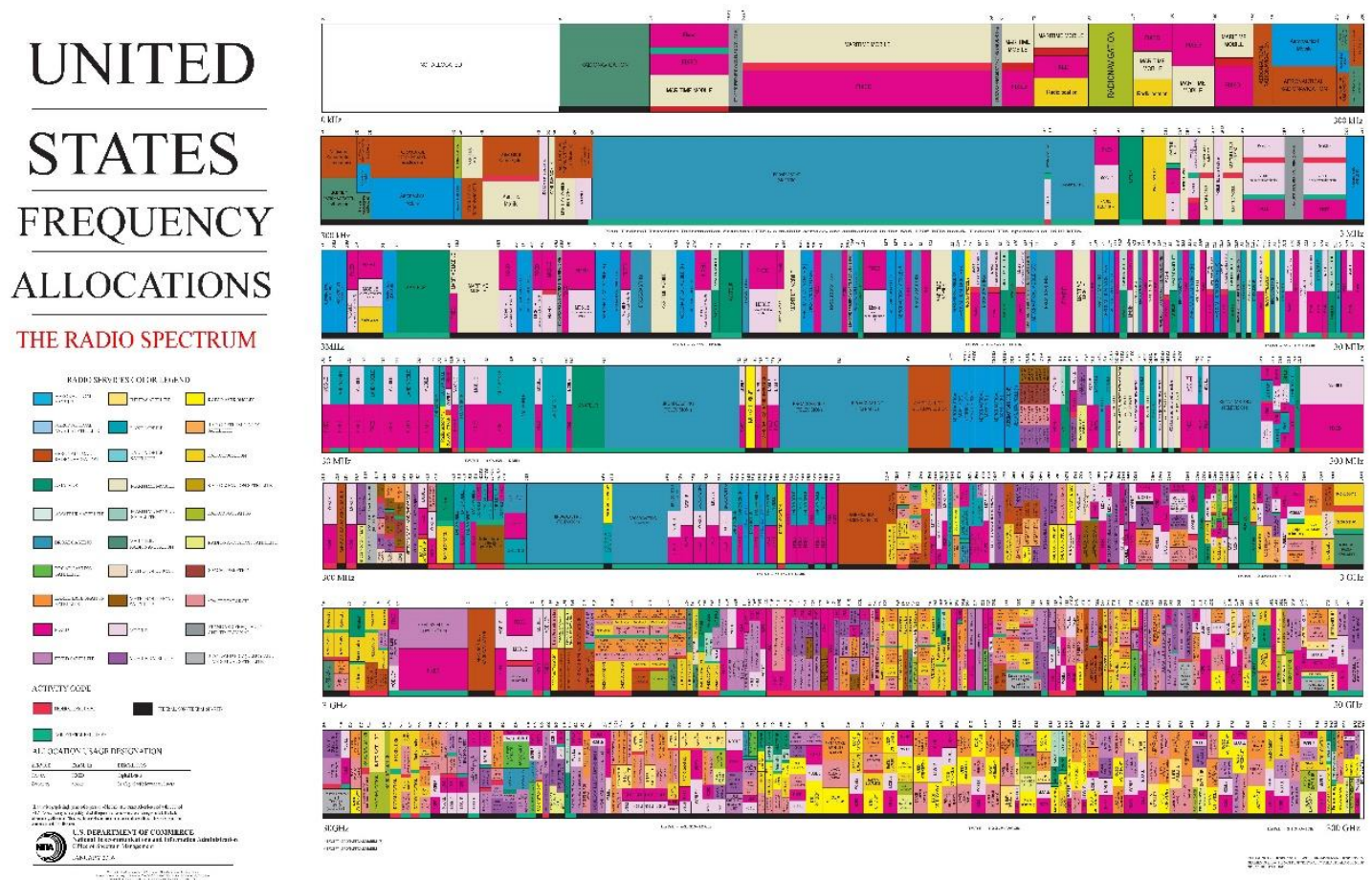

Figure 2: US allocation chart of 2011 


\subsection{Methodology}

The Nigeria frequency allocation table sets out all allocations made from $8.3 \mathrm{kHz}$ to $300 \mathrm{GHz}$. The table is presented in six segments with each segment having five columns (Commission \& others, 2021). The allocated spectrum bands are classified into six ranges where each segment represents a range of frequencies as follows:

i) Segment 1: Below $30 \mathrm{MHz}$

ii) Segment 2: 30-300MHz

iii) Segment 3: $300 \mathrm{MHz}$ to $3 \mathrm{GHz}$

iv) Segment 4: 3-10GHz

v) Segment 5: $10-30 \mathrm{GHz}$

vi) Segment 6: 30-300GHz

In each segment of this table, five columns are used to portray the following:

i) Frequency bands

ii) ITU Region 1 allocations

iii) Nigerian allocations

iv) Nigerian utilization

v) Remarks

Figure 3 shows some entries of the frequency allocation table of Nigeria.

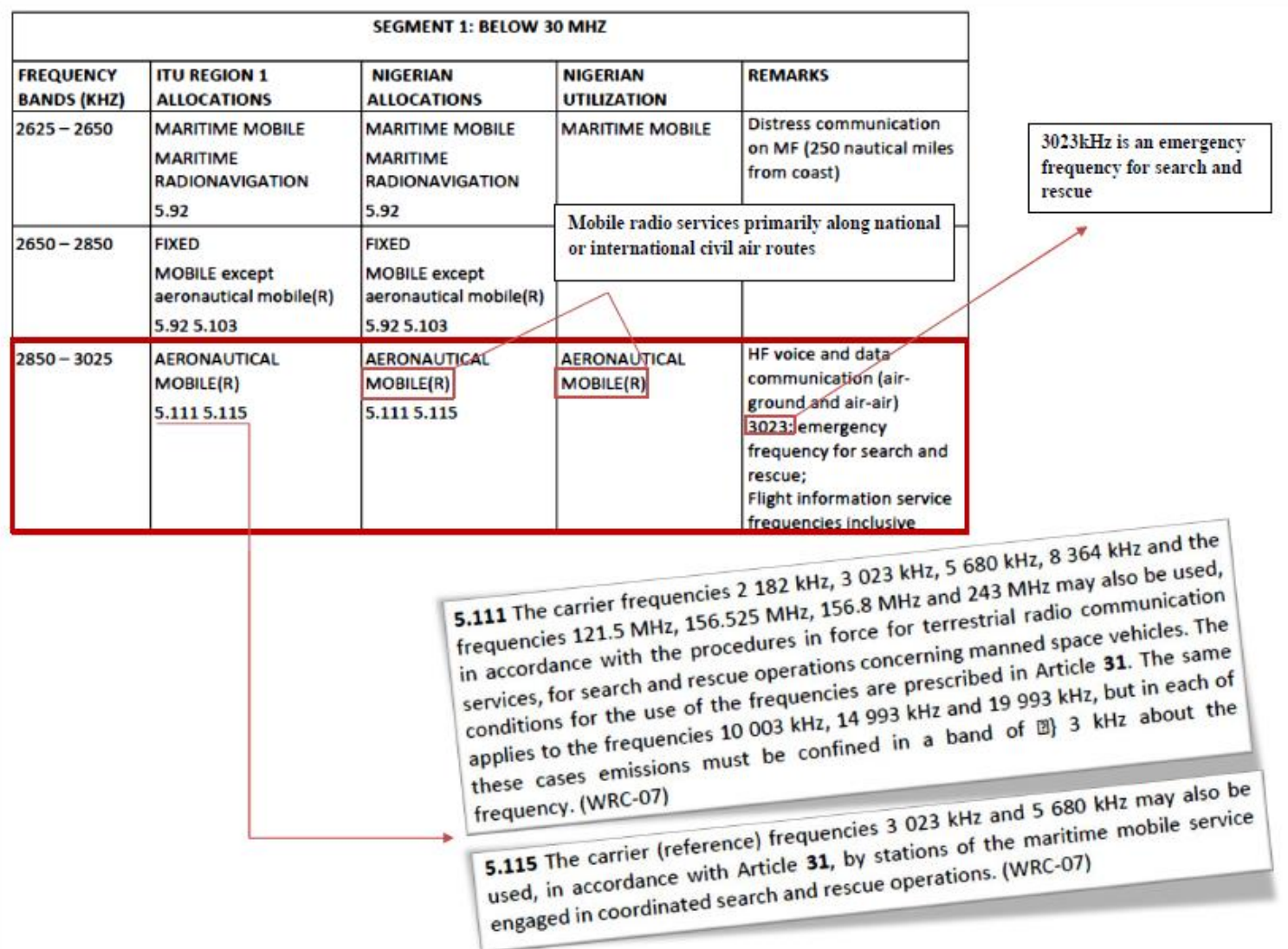

Figure 3: Table entries of the Nigeria frequency allocation table

The first column gives the allocated bands, for instance, 2850 - 3025 are the frequency bands in $\mathrm{MHz}$ allocated to one or two services depending on the conditions for the use of the allocated band. The second column is the allocations given by ITU to region 1 . The next column after it is the column of interest that contains the allocations in Nigeria. It is either a replica of the recommended allocations by ITU or slightly changed based on some guidelines. The highlighted row shows the allocations for Aeronautical Mobile primarily along national or international civil air routes. This is signified as Aeronautical Mobile (R). Lastly, the remarks column gives extra information on frequencies in the 
allocated band. For instance, the frequency $3023 \mathrm{kHz}$ in the allocated band is an emergency frequency for search and rescue. Footnotes often specify more information about the condition of use of the allocated band of frequencies. The footnote in the figure reiterates the information in the remark stating again that $3023 \mathrm{kHz}$ may be used for search and rescue alongside some other frequencies.

The chart accompanying this article shows how frequency is allocated in Nigeria as contained in the NTFA. The list of radio services in the NTFA are the Aeronautical Mobile, Aeronautical Mobile primarily along national and international civil air routes, Aeronautical Mobile primarily outside national and international civil air routes, Aeronautical Mobile Satellite, Aeronautical Radionavigation, Amateur, Amateur Satellite, Broadcasting, Broadcasting Satellite, Earth Exploration Satellite, Fixed, Fixed Satellite, Inter Satellite, Land Mobile, Maritime Mobile, Maritime Radionavigation, Meteorological aids, Meteorological Satellite, Mobile, Mobile except for aeronautical mobile, Mobile except for aeronautical mobile majorly along national and international civil air routes, Mobile Satellite, Not Allocated, Radio Astronomy, Radiolocation, Radionavigation, Radionavigation Satellite, Space Operation, Space Research, Standard Frequency and Time signal, and Standard Frequency and Time signal Satellite. These radio services are assigned band of frequencies in the radio spectrum. Each frequency band can contain two or more services having equal or unequal rights. To achieve a comprehensive spectrum allocation chart, the radio spectrum allocations were split into 8 bands that start from the very low frequency (VLF) band to the extremely high frequency (EHF) band; these bands and their respective frequency ranges are presented in Table 1. In each of these bands, the radio services were then constructed as small quadrilaterals vector shapes which are stacked vertically in their frequency allocations to make up the spectrum allocation chart. To distinguish the different radio services the vector shapes had colours assigned to them for the respective radio services they represent. Also, the different rights (primary and secondary users) were distinguished using spot patterns, according to the information in the NTFA and all exceptions were represented using letters whose meanings are contained in the legend. Important remarks were also added as labels pointing to the frequency band that holds the information. The frequency allocations in the chart were not designed to scale and the chart was designed with Photoshop.

Table 1: Frequency allocation bands in the RF spectrum

\begin{tabular}{lll}
\hline Frequency band & Symbol & Frequency range \\
\hline Very low frequency & VLF & $3 \mathrm{kHz}$ to $30 \mathrm{kHz}$ \\
Low Frequency & $\mathrm{LF}$ & $30 \mathrm{kHz}$ to $300 \mathrm{kHz}$ \\
Medium Frequency & $\mathrm{MF}$ & $300 \mathrm{kHz}$ to $3 \mathrm{MHz}$ \\
High frequency & $\mathrm{HF}$ & $3 \mathrm{MHz}$ to $30 \mathrm{MHz}$ \\
Very High Frequency & $\mathrm{VHF}$ & $30 \mathrm{MHz}$ to $300 \mathrm{MHz}$ \\
Ultra High Frequency & $\mathrm{UHF}$ & $300 \mathrm{MHz}$ to $3 \mathrm{GHz}$ \\
Super High Frequency & $\mathrm{SHF}$ & $3 \mathrm{GHz}$ to $30 \mathrm{GHz}$ \\
Extremely High Frequency & $\mathrm{EHF}$ & $30 \mathrm{GHz}$ to $300 \mathrm{GHz}$ \\
\hline
\end{tabular}

The spectrum chart was accomplished by using vector shapes to allow high zoom capabilities. Photoshop (Photoshop, 2017) is an image editing program that can open an image and help create new documents, including vector graphics - scalable image files. It is used to edit images with its comprehensive set of retouching, painting, and drawing tools. Every project in Photoshop starts with some settings, most times the default settings are good for a lot of designs, but it is worth the mention. Some notable settings a designer can tweak are the canvas size which can be chosen from a pre-set collection or simply customized, also there is the resolution and the colour mode (RGB, CMSK, or Grayscale). Particularly, the designer can change the resolution to suit printing size. Table 2 contains the setting used for the design of the spectrum allocation chart.

Table 2: Project settings for the spectrum allocation chart

\begin{tabular}{llll}
\hline Project settings & Resolution & Canvas Size & Colour mode \\
\hline Values & 300 pixels/inches & 14 by 8.5 inches & RGB colour \\
\hline
\end{tabular}

\subsection{Results and Discussion}

Figure 4 is the result of the detailed design of the spectrum allocation chart. It shows eight bands ranging from the very low frequency (VLF) to the extremely high frequency (EHF) band. There are 20 different colours for the radio services including the Industrial, Scientific and Medical (ISM) band. 
The frequencies are labelled at the top of each band alongside the remarks for the respective frequency bands and it should be noted that the splitting of the allocated bands is not to scale.

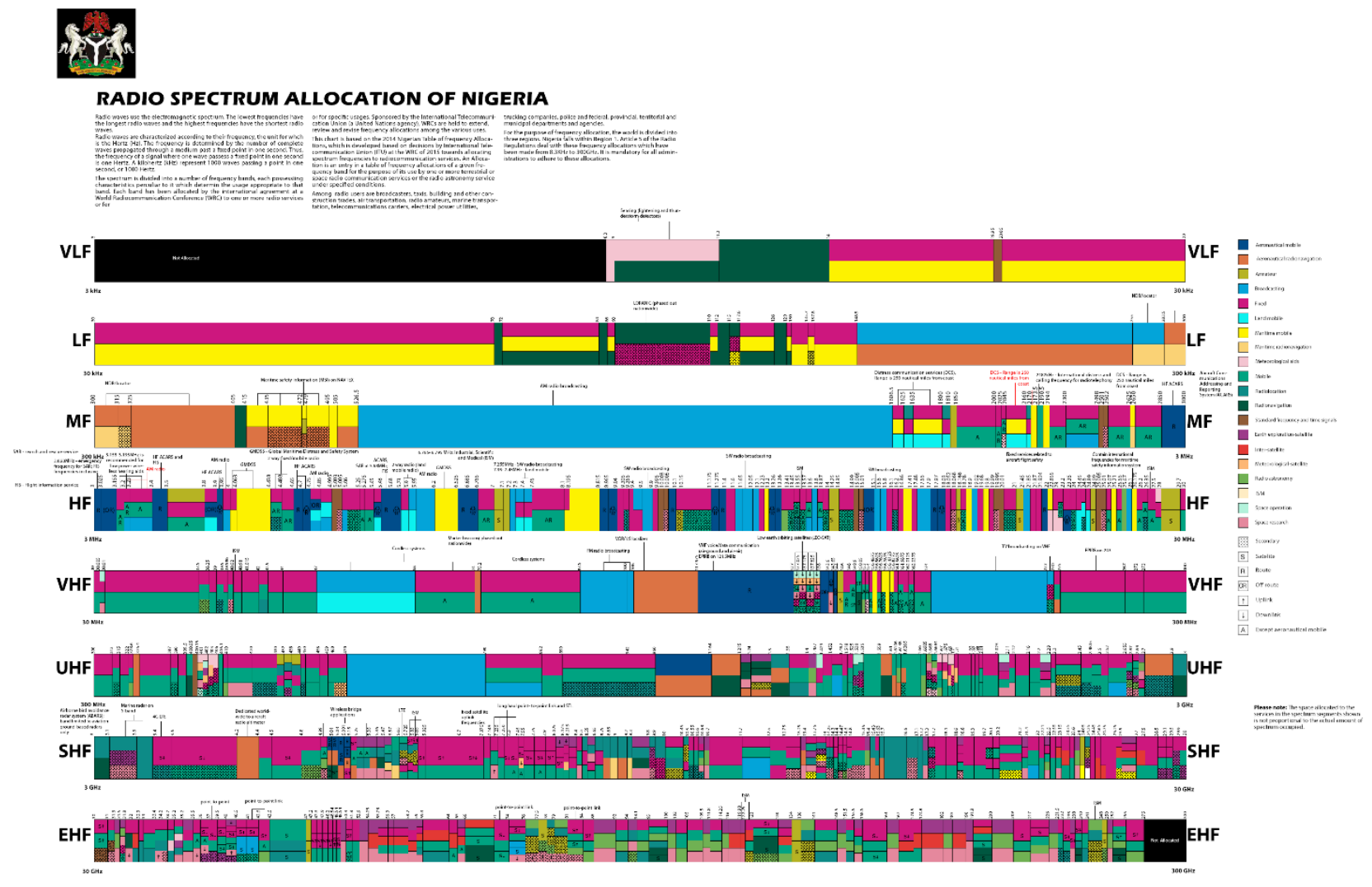

Figure 4: Nigeria frequency allocation chart

The allocation chart concisely and graphically represents the information across all of the radio services operating in Nigeria contained in the NTFA. It was observed that the allocations made in the NTFA complied with the recommendation for the ITU region 1 and that all the frequencies between $8.3 \mathrm{kHz}$ and $300 \mathrm{GHz}$ were allocated with the exception of the frequency band 275 to $300 \mathrm{GHz}$. It was also observed that there are more allocations in the higher frequency bands than the lower frequency bands. Moreover, fixed and mobile radio services had the most frequency allocations compared to the other radio services in the spectrum allocation. However, although most of the commercial frequencies have been allocated, the utilization percentage of the radio spectrum is not commensurate. From the NTFA, it was observed that most of the allocated bands where not been used by the radio services assigned to them. This finding correlates with the increasing findings from several researchers on the issue of spectrum underutilization where there is the problem of spectrum scarcity. Researchers have found a low spectrum usage in their researches (Islam, et al., 2008; Muñoz Montero, 2014; Alonso, et al., 2020; Sayyed, et al., 2021) and this observation can be inferred in Nigeria with the low utilization from the assigned radio services that was recorded in the NTFA of Nigeria.

\subsection{Conclusion}

This study fully analysed the radio frequency management standards and polices in two Englishspeaking countries, the UK and the USA, which are leading countries in the world. This analysis has provided an overview of the subject area using the selected countries as a case study. Also, the study produced the spectrum allocation chart of Nigeria in a document of high-zoom capabilities which is suitable for public consumption and spectrum allocation overview.

\section{References}

Alonso, R. M. et al., (2020). Assessment of White Spaces Quality in Rural Areas: a large-scale spectrum survey. s.l., s.n., pp. 1-5. 
Ancans, G., Bobrovs, V. and Ivanovs, G. (2013). Spectrum Usage in Mobile Broadband Communication Systems/RADIOFREKVENČU IZMANTOŠANA MOBILO PLATJOSLAS SAKARU SISTĒMĀS. Latvian Journal of Physics and Technical Sciences, 50, pp. 49-58.

Brinson, S. L. (2004). The Red Scare, Politics, and the Federal Communications Commission, 19411960. s.l.: Greenwood Publishing Group.

Bulmer, S. (2020). The member states of the European Union. s.1.:Oxford University Press, USA.

Commission, F. C. \& others, 2021. FCC. [Online]

Available at: https://www.fcc.gov/

Commission, N. C. \& others, 2021. Nigerian Communications Commission. [Online]

Available at: https://www.ncc.gov.ng/

Commission, N. C. \& others, 2021. Regulations. [Online]

Available at: https://www.ncc.gov.ng/licensing-regulation/legal/regulations

Data, B. A., 1994. National Telecommunications and Information Administration.

DAU, 2013. Spectrum and E3 Compliance. [Online]

Available at: https://www.dau.edu/cop/e3/Pages/SPDP037.aspx

DCS (2014). The UK spectrum strategy. Minister for Culture, Communications and Creative Industries.

Doyle, G. and Vick, D. W. (2005). The Communications Act 2003: A new regulatory framework in the UK. Convergence, 11, pp. 75-94.

Ilcev, S. D. (2005). Global Mobile Satellite Communications: For Maritime, Land and Aeronautical Applications. s.l.: Springer Science \& Business Media.

Islam, M. H. et al., (2008). Spectrum survey in Singapore: Occupancy measurements and analyses. s.1., s.n., pp. 1-7.

Mawoli, M. (2009). Liberalisation of the Nigerian telecommunication sector: a critical review. Journal of Research in National Development, 7.

Mazar, H. (2016). Radio spectrum Management: Policies, regulations and techniques. s.l.:John Wiley \& Sons.

McLoughlin, G. J. (2005). The National Telecommunications and Information Administration (NTIA): Budget, Programs, and Issues. s.l., s.n.

Muñoz Montero, Ó. (2014). Spectrum survey in sub-urban environment: Campus of Universitat Autònoma de Barcelona.

Photoshop, A. (2017). Adobe Photoshop. Red, 2, p. 168.

Research, R. M. (2018). The Roke United Kingdom Frequency Allocation Table. [Online] Available at: https://www.roke.co.uk/expertise/sensors-and-communications/the-roke-unitedkingdom-frequency-allocation-table

Sayyed, M. I., Naikwadi, M. M. H. and Sagat, M. S. P. (2021). FREQUENCY SPECTRUM MEASUREMENT AND OCCUPANCY ANALYSIS.

Telecomm, N. and Admin, I. (2011). US frequency allocation chart as of 2011. s.1.:s.n.

Union, I. T., 2021. ITU. [Online] Available at: https://www.itu.int 
Uzairue, S. I. et al., (2018). A Review: The past, present and future of radio frequency spectrum in Nigeria, Canada, United Kingdom, Ghana. International Research Journal of Engineering and Technology (IRJET), 6, pp. 1034-1039. 